\title{
THE SOLAR MASS EJECTION IMAGER (SMEI) SPACE EXPERIMENT
}

Richard R. Radick

30 January 2015

Final Report

APPROVED FOR PUBLIC RELEASE; DISTRIBUTION IS UNLIMITED.

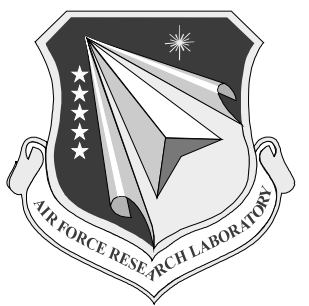

AIR FORCE RESEARCH LABORATORY Space Vehicles Directorate 3550 Aberdeen Ave SE AIR FORCE MATERIEL COMMAND KIRTLAND AIR FORCE BASE, NM 87117-5776 


\section{DTIC COPY}

\section{NOTICE AND SIGNATURE PAGE}

Using Government drawings, specifications, or other data included in this document for any purpose other than Government procurement does not in any way obligate the U.S. Government. The fact that the Government formulated or supplied the drawings, specifications, or other data does not license the holder or any other person or corporation; or convey any rights or permission to manufacture, use, or sell any patented invention that may relate to them.

This report was cleared for public release by the 377 ABW Public Affairs Office and is available to the general public, including foreign nationals. Copies may be obtained from the Defense Technical Information Center (DTIC) (http://www.dtic.mil).

AFRL-RV-PS-TR-2014-0197 HAS BEEN REVIEWED AND IS APPROVED FOR

PUBLICATION IN ACCORDANCE WITH ASSIGNED DISTRIBUTION STATEMENT.

//SIGNED//

Dr. Karatholuvu S. Balasubramaniam

Project Manager, AFRL/RVBXS
//SIGNED//

Glenn M. Vaughan, Colonel, USAF

Chief, Battlespace Environment Division

This report is published in the interest of scientific and technical information exchange, and its publication does not constitute the Government's approval or disapproval of its ideas or findings. 


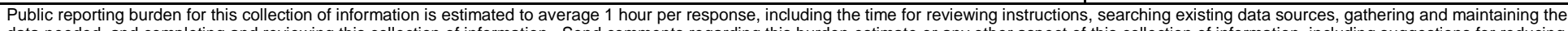

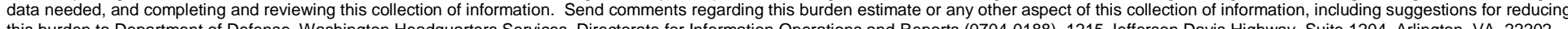

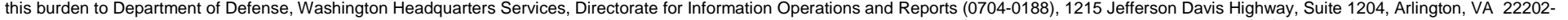

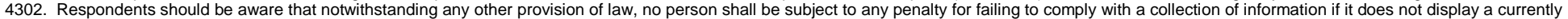
valid OMB control number. PLEASE DO NOT RETURN YOUR FORM TO THE ABOVE ADDRESS.

\begin{tabular}{l|l|l} 
1. REPORT DATE (DD-MM-YYYY) & 2. REPORT TYPE & 3. DATES COVERED (FrOm - To)
\end{tabular}

30-01-2015

Final Report

4. TITLE AND SUBTITLE

The Solar Mass Ejection Imager (SMEI) Space Experiment

01 Apr 2007 to 06 Aug 2014

5a. CONTRACT NUMBER

5b. GRANT NUMBER

5c. PROGRAM ELEMENT NUMBER

$63401 \mathrm{~F}$

6. AUTHOR(S)

Richard R. Radick

5d. PROJECT NUMBER

5021

5e. TASK NUMBER

PPM00004258

5f. WORK UNIT NUMBER

EF004416

\section{PERFORMING ORGANIZATION NAME(S) AND ADDRESS(ES)}

8. PERFORMING ORGANIZATION REPORT NUMBER

Air Force Research Laboratory

Space Vehicles Directorate

AFRL-RV-PS-TR-2014-0197

3550 Aberdeen Avenue SE

Kirtland AFB, NM 87117-5776

9. SPONSORING I MONITORING AGENCY NAME(S) AND ADDRESS(ES)

10. SPONSOR/MONITOR'S ACRONYM(S)

AFRL/RVBXS

11. SPONSOR/MONITOR'S REPORT

NUMBER(S)

\section{DISTRIBUTION / AVAILABILITY STATEMENT}

Approved for public release; distribution is unlimited. (377ABW-2015-0172 dtd 02 Mar 2015)

\section{SUPPLEMENTARY NOTES}

\section{ABSTRACT}

The Solar Mass Ejection Imager (SMEI) space experiment was an operational prototype for a new class of white light heliospheric imager. SMEI was launched on the Coriolis spacecraft in January, 2003, and was deactivated in September, 2011, thus operating almost continuously for more than eight years. Its primary objective was the detection and tracking of interplanetary transients, typically coronal mass ejections (CMEs), in the inner heliosphere. Towards this goal it was immediately effective, observing and tracking several CMEs in the first month of mission operations, with almost 400 detections to follow. SMEI also contributed to many and varied scientific fields, including studies of corotating interaction regions, the high-altitude aurora, zodiacal light, Gegenschein, comet tail disconnections and motions, and variable stars. It was also able to detect and track Earth-orbiting satellites and space debris. Along with its scientific achievements, SMEI also enabled a significantly improved ability to forecast the arrival of CMEs at Earth, thereby establishing the operational utility of heliospheric imaging. This report reviews the SMEI mission and instrument, its scientific and technical accomplishments, and provides a summary discussion of potential next steps for future heliospheric imagers.

\section{SUBJECT TERMS}

Solar Mass Ejection Imager, SMEI, Heliospheric Imager, Coronal Mass Ejections, CMEs

16. SECURITY CLASSIFICATION OF:

\section{a. REPORT}

Unclassified

\section{b. ABSTRACT}

Unclassified
17. LIMITATION OF ABSTRACT

Unlimited

18. NUMBER
OF PAGES
32

19a. NAME OF RESPONSIBLE PERSON

Dr. K. S. Balasubramaniam

19b. TELEPHONE NUMBER (include area code) 
This page is intentionally left blank.

Approved for public release; distribution is unlimited. 


\section{Table of Contents}

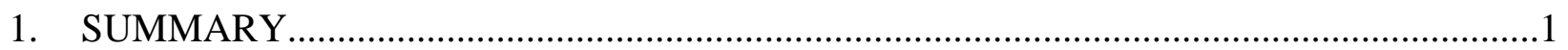

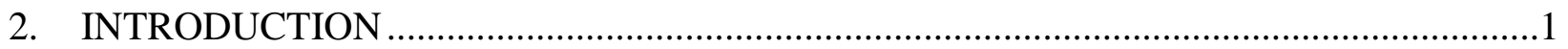

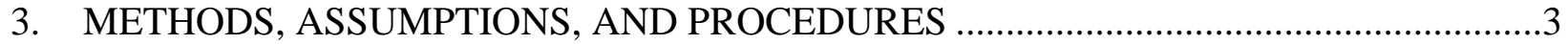

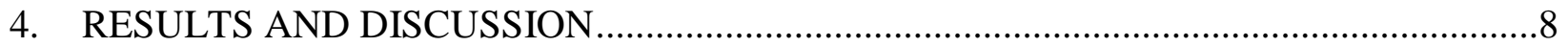

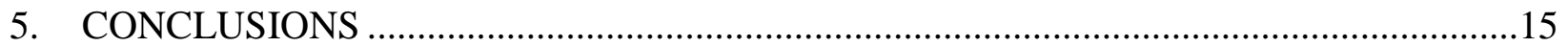

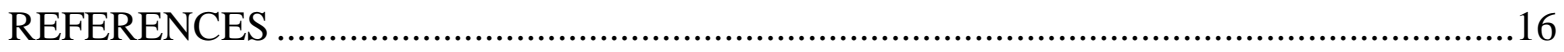


This page is intentionally left blank. 


\section{SUMMARY}

The Solar Mass Ejection Imager (SMEI) space experiment was an operational prototype for a new class of white light heliospheric imager. A heliospheric imager (HI) observes sunlight that has been Thomson-scattered by free electrons in the solar wind. Compared with traditional coronagraphs, an HI observes at much larger angles from the Sun, and hence requires a much higher sensitivity and wider dynamic range. SMEI was launched on the Coriolis spacecraft in January, 2003, and was deactivated in September, 2011, thus operating almost continuously for more than eight years. Its primary objective was the detection and tracking of interplanetary transients, typically coronal mass ejections (CMEs), in the inner heliosphere. Towards this goal it was immediately effective, observing and tracking several CMEs in the first month of mission operations, with almost 400 detections to follow. Along with this primary science objective, SMEI also contributed to many and varied scientific fields, including studies of corotating interaction regions (CIRs), the high-altitude aurora, zodiacal light, Gegenschein, comet tail disconnections and motions, and variable stars. It was also able to detect and track Earth-orbiting satellites and space debris. Along with its scientific achievements, SMEI also enabled a significantly improved ability to forecast the arrival of CMEs at Earth, thereby establishing the operational utility of heliospheric imaging. In this report we briefly discuss the SMEI mission and instrument, review its scientific and technical accomplishments, and provide a summary discussion of potential next steps for future HIs.

\section{INTRODUCTION}

The Solar Mass Ejection Imager (SMEI) space experiment [1, 2] was launched on 6 January 2003 aboard the Coriolis spacecraft. SMEI served as a prototype and proof-of-concept for a new class of visible white light imager for heliospheric observations.

The heliosphere is the volume of space surrounding the Sun that is dominated by the solar wind; the Earth is embedded deep within the heliosphere. A heliospheric imager (HI) observes sunlight that has been Thomson-scattered by free electrons in the solar wind. HIs have much higher sensitivity and greater dynamic range than conventional coronagraphs, enabling them to observe the heliosphere to much larger angular distances from the Sun. Subsequent to SMEI, HIs are now on board the twin spacecraft of NASA's ongoing Solar Terrestrial Relations Observatory (STEREO) mission [3, 4]. SMEI itself operated almost continuously for over 8-1/2 years until its deactivation in September, 2011, observing almost the entire heliosphere outwards from about $18^{\circ}$ away from the Sun.

Heliospheric imaging dates back to the Helios mission [5], whose zodiacal light experiment observed interplanetary transients in white light at large distances from the Sun. Although over 20 years elapsed from the end of the Helios mission in 1982 to the launch of SMEI in 2003, the SMEI concept dates back to the Helios era and was a direct outgrowth of that mission [6].

The primary objective of SMEI was the detection and tracking of interplanetary transients, specifically coronal mass ejections (CMEs), as they travel through the heliosphere from the Sun 
to the Earth and beyond. Towards this objective it was immediately successful, with several CMEs observed during its first month of mission operations [7]. A further objective was to forecast the time of arrival of CMEs at Earth. To this end SMEI was used to measure distances of heliospheric structures from Earth prior to their Earth arrival. SMEI also provided useful information to other scientific fields, including high-altitude aurorae, the zodiacal light, the Gegenschein, variable stars, and comets. The SMEI experiment has produced over 113 peerreviewed papers, 195 other post-launch articles and conference proceedings, and 32 press releases or public articles. These numbers continue to increase.

Coronal mass ejections $[\mathbf{8}, \mathbf{9}]$ are large bubbles of magnetized plasma that are ejected from the Sun's outer atmosphere, or corona, over the course of several hours and subsequently propagate outward through interplanetary space. CMEs are formed by the reconfiguration of large-scale coronal magnetic fields during a magnetic reconnection process that is not yet fully understood. CMEs are launched with a wide range of speeds, from less than $10 \mathrm{~km}$ per second to more than 2,000 km per second. Fast CMEs are those that travel faster than the background solar wind, which has a typical speed of about $400 \mathrm{~km}$ per second. Fast CMEs can drive interplanetary shocks that disrupt the normal flow of the solar wind.

If the magnetic field inside a CME arriving at Earth has a strong southward component, it can efficiently couple with the northward-pointing geomagnetic field, thereby transferring mass and energy from the solar wind into the magnetosphere and generating a geomagnetic storm. In addition to the strong, non-recurrent geomagnetic storms caused by CMEs, there is a second class of generally weaker recurrent geomagnetic storms associated with long-lived features on the Sun, called coronal holes, that generate co-rotating interaction regions (CIRs) in the solar wind when the fast solar wind from the coronal holes catches up with the slow solar wind. Recurrent geomagnetic storms tend to repeat with the 27-day solar rotation period.

The main drivers of space weather, geomagnetic storms are disturbances in Earth's magnetosphere that can have significant impact on both ground- and space-based technological systems. The largest geomagnetic storm ever recorded, that of September 2, 1859, produced widespread disruption of telegraphic communications along with intense auroral displays as far south as Cuba [10]. On the previous day, astronomer Richard Carrington of the Royal Greenwich Observatory had made the first-ever observation of a white-light solar flare. Carrington noted the coincidence (but did not claim a direct connection) between the aurorae and the solar flare.

Although CMEs are often associated with large solar flares and with prominence eruptions, they can also occur in the absence of either of these other two processes. The frequency of CMEs varies with the 11-year solar activity cycle. At solar minimum, CMEs occur at the rate of about one per week. Near solar maximum, they can occur at a rate of two or three per day. The energy carried in a fast CME is approximately the same as that released in a large solar flare.

CMEs were first discovered from observations made with a coronagraph aboard the OSO-7 spacecraft in the early 1970s [11]. A coronagraph is a special telescope that produces an artificial eclipse of the Sun. During a natural eclipse, caused by the Moon passing in front of the Sun, the solar corona is visible at most for only a few minutes during totality, too short to notice changes in coronal features associated with CMEs. With ground-based coronagraphs, only the very 
innermost corona is visible above the brightness of the sky, and not the outer corona where CMEs occur. From space, however, the corona is visible out to large distances from the Sun and can be viewed continuously. With the advent of space-based solar coronagraphs, high-resolution and relatively continuous observations of the Sun's corona can be made, allowing for the routine observation of CMEs. The Large Angle and Spectrometric Coronagraph (LASCO) aboard the Solar and Heliospheric Observatory (SOHO) [12] has been observing CMEs since its launch in 1995, and remains the only source of such observations in operation today.

In this report we briefly discuss the SMEI mission and instrument, review its scientific and technical accomplishments, and provide a summary discussion of potential next steps for a future heliospheric imager. A comprehensive description of the SMEI mission [13], written by the SMEI science team and published in 2013 in Space Science Reviews, should be consulted for additional detail on any of these topics.

\section{METHODS, ASSUMPTIONS, AND PROCEDURES}

\subsection{The SMEI Instrument and Mission}

SMEI was launched on board the Air Force Space Test Program's Coriolis spacecraft from Vandenberg Air Force Base on 6 January 2003. It was one of two instruments aboard Coriolis, the primary payload being the Naval Research Laboratory's WindSat microwave polarimetry experiment [14] to measure ocean surface wind speed and direction. Coriolis was inserted into a dawn-dusk, Sun-synchronous polar orbit, inclined at $98^{\circ}$ from the equator at an altitude of 840 $\mathrm{km}$ and a 102-min period. The spacecraft was nadir/velocity-vector stabilized.

The SMEI project was a partnership among the Air Force Research Laboratory (AFRL), the University of Birmingham (UB) in the United Kingdom, and the University of California - San Diego (UCSD). UCSD contributed the optical design and scientific \& technical expertise; UB designed \& built the SMEI instrument, supported spacecraft integration, and participated in mission operations; AFRL provided overall project management and co-ordination with the Space Test Program, served as project lead for mission operations, and managed data ground distribution and processing. The three partners shared the project science. NASA's Earth-Sun Systems Division provided partial funding for SMEI via awards to AFRL.

The SMEI instrument consisted of three wide-angle, white light (450-950 nm), 1280 x 576 pixel CCD cameras and a central Data Handling Unit (DHU) that controlled the readout of the cameras and provided the command and telemetry data interfaces with the spacecraft [15]. Each camera was provided with a multi-stage labyrinthine baffle that, in conjunction with the camera optics, reduced stray light striking the detector to $1 \times 10^{-15}$, relative to the Sun's brightness [16], an unprecedented amount. This amount of attenuation is required to observe CMEs, which are very large but also intrinsically very, very faint.

Each of the cameras imaged, with a 4-second exposure, a $3^{\circ} \times 60^{\circ}$ strip of sky with a $0.05^{\circ}$ detector pixel scale. The cameras were deliberately defocused to an angular resolution of about $0.5^{\circ}$ to help achieve a photometric precision of $0.1 \%$ across the field-of-view (FOV); for 
observing CMEs, sensitivity and uniform precision across the FOV are more important than angular resolution. The cameras were mounted on the spacecraft to sweep through, once per orbit, three somewhat overlapping, $60^{\circ}$-wide arcs of sky around the pitch axis of the zenith-nadir pointed Coriolis spacecraft. The frame-store operation of the CCD cameras enabled exposures with minimal gaps between frames. This meant that almost the entire sky was observed during each 102-minute orbit, excepting an $18^{\circ}$-radius zone in the nominally sunward direction of the pitch axis and a much smaller zone in the nominally anti-solar direction. The sunward exclusion zone was required because the baffle would have been unable to reject sunlight adequately had the sunward-facing camera (“Camera 3”) been pointed more directly toward the Sun.

For most of the year, apart from a short period close to the summer solstice, the Sun lay within the $18^{\circ}$-radius exclusion zone of Camera 3. However, when the Sun approached closer than about $7^{\circ}$ from the edge of that camera's FOV, a protective shutter was closed to prevent sunlight from reaching the CCD. On average, this closure reduced the sky coverage for Camera 3 to about $60 \%$ of the maximum possible. During normal operations the shutters of the other two cameras did not close.

Early flight data confirmed that the stray-light rejection specification for SMEI was achieved over most of the FOV, and that the photometric specification was met for the majority of the sky for two of the three cameras. The performance of the Camera 3, with its higher noise level, was relatively poor, and grew progressively worse throughout the mission - this will be discussed at greater length subsequently.

The protective doors of the three cameras were opened on 1 February 2003; the first day of usable observations was 5 February; and the first complete day of science observations was on 6 February. The first CME was observed on 10 February, and 11 more were detected by the end of that first month.

SMEI operated almost continuously from early February 2003 through late September 2011, excepting three gaps that occurred in 2005, 2006 and 2007. The 2005 and 2007 gaps were caused by the Coriolis spacecraft transitioning to safe mode, in the former case as a result of a WindSat anomaly and in the latter case due to problems with the Coriolis's star tracker. The 2006 gap was caused by the only significant hardware failure on SMEI during the mission. The "A"-side of the DHU failed during a passage of Coriolis through the South Atlantic Anomaly (SAA), requiring that control of the instrument be transferred to the "B"- side DHU. Passages through regions of high particle background (SAA and polar regions) caused regular processor anomalies in the DHU, typically every few weeks throughout the mission: each time required a processor re-boot for recovery. Prior to 2008 this was handled entirely manually, resulting in delays of some hours in restoring observations. However, in early 2008 new procedures were introduced, allowing the operations team to reboot SMEI without consulting the SMEI team for well-understood anomalies. This resulted in a much faster recovery following anomalies. Therefore, operations between 2008-2011 were relatively routine \& continuous.

Processing of the SMEI images involved projecting the individual camera exposures back onto the sky to form a composite sky map. Because each SMEI sky map is a built-up "image" assembled from a series of exposures, every location on the map corresponds to a slightly 
different time throughout the orbit. A SMEI sky map is, therefore, not a "still" frame, such as is acquired from the heliospheric imagers aboard STEREO or most coronagraphs, but rather a timevarying scan across a 102-minute orbit. The short time that SMEI viewed a given location in the sky created sharper images of transients compared with the more conventional staring optical systems. This feature enabled more accurate measurements of their features (e.g., their leading edges). However, because a whole SMEI sky map does not provide an average at a single instant in time, the corresponding motion of heliospheric structures must properly be taken into account to calculate accurate structure locations.

SMEI was deactivated on 28 September 2011 after 8-1/2 years in operation. As of early 2015, Coriolis and WindSat remain operational. Presumably, SMEI could be re-activated if resources were available to support mission operations and the data processing efforts.

\subsection{Factors Affecting SMEI Image and Sky Map Quality}

The SMEI cameras required a high sensitivity and wide dynamic range in white light, as CMEs and other heliospheric structures such as CIRs have an intensity of at most a few $\times 10^{-14}$ times the mean surface brightness of the Sun. Moreover, since the surface brightness of these features rapidly diminishes with distance from the Sun, the dynamic range of the instrument must span at least four decades. Furthermore, the cameras had sources of background and other factors affecting image quality, including instrumental (hot pixels, glare, scattered light), environmental (particle impacts during the SAA and auroral oval passages), and astronomical (Moon, planets, zodiacal light, individual stars and galactic background).

3.2.1 Instrumental Factors. Significant instrumental noise was caused by "hot pixels". These are CCD pixels that show a significantly-elevated dark current and in some cases may flip between normal and hot states. This was particularly problematic in Camera 3, which was operating at temperatures typically between $-15^{\circ} \mathrm{C}$ and $0^{\circ} \mathrm{C}$ (depending on the time of year), substantially above the design temperature of $-30^{\circ} \mathrm{C}$. This caused many pixels to be hot, and some flipped on timescales of less than an hour. The other two cameras, which operated close to or below the design temperature, also developed some hot pixels later in the mission, but these were not sufficiently numerous to noticeably impact their performance.

Annealing returned many of the hot pixels to their normal state. For this process, the CCDs were typically heated to about $+60^{\circ} \mathrm{C}$ to $+80^{\circ} \mathrm{C}$ for a few hours, using the decontamination heaters that were closely coupled thermally to the CCD chip package. Because of its numerous hot pixels, Camera 3 was annealed more often than the other two and at a higher temperature $\left(+80^{\circ} \mathrm{C}\right)$. Despite these annealing efforts, hot pixels gradually accumulated throughout the mission, contributing to the dark charge noise and degrading the image quality. By around 2006 Camera 3 images had degraded to the point where only the brightest transients could be easily detected, and by the end of the mission in 2011 a second camera had also noticeably degraded, but not enough to compromise its ability to observe CMEs. Each camera displayed an increase in dark charge through the course of the mission due to orbital radiation flux, but the most significant degradation by far was in Camera 3. Nonetheless, we note that SMEI operated well 
beyond its nominal design life of 2 years, and it also exceeded the 7 year design life of the spacecraft.

A second contribution from the instrument was "glare." This is light reaching the CCD image-plane via a complicated, unblocked scattering path through the baffle and camera optics. This light could be modeled and thus largely eliminated from the final SMEI maps.

3.2.2 Environmental Factors. The polar Earth-orbit of Coriolis produced additional sources of noise, primarily from energetic particles striking the CCD chips. The two most troublesome orbital regions for this were the SAA, which is essentially an extension of the Van Allen radiation belt to low altitudes, and the auroral-oval zones, where the ionosphere is directly exposed to energetic particle precipitation from the solar wind. When especially numerous, energetic particles create the well-known "snow storm" effect that is common with coronagraph images (e.g., LASCO) as energetic solar particles impact the instrument. In the auroral zones the particles were most likely to be electrons: these were first collimated by the baffles and then scattered by the main mirror in the SMEI optics to reach the CCD directly. In the SAA the particles were mostly energetic protons that penetrated the instrument housing directly. At low to moderate fluxes, the affected CCD pixels were identified in the SMEI analysis and excluded from the summing to make the final maps, but with high fluxes this was no longer possible.

3.2.3 Astronomical Factors. SMEI was capable of detecting stars down to 10th magnitude. Consequently, stars dominated the field of each camera. In principle, these could be individually subtracted from each sky map, or even from individual data frames before forming a sky map. In practice, only stars brighter than 6th magnitude were individually subtracted, since SMEI's large point-spread function (PSF) causes significant overlap when dealing with the increasingly numerous stars fainter than this.

Saturation by the Sun in the Camera 3 image occurred when the spacecraft attitude was such that the Sun's location approached too closely to its FOV. Closing the shutter removed some, but not all, of this. Another large, bright feature is the Moon, which posed a significant problem for SMEI observations, as it completely saturated large portions of the sky even when it was only a narrow crescent. While nothing could be done to remove the saturation effects of the Moon, it was a known and predictable feature, easily flagged as bad data. Likewise, the brighter planets Venus and Jupiter saturated SMEI in a predictable manner and so they could also be flagged, while the other planets could be treated as "stars", and subtracted.

The final source of astronomical background was the zodiacal light, also known as the Fcorona. For SMEI this light dominated the sky, particularly close to the Sun around the ecliptic plane. When observed at the sensitivity of SMEI, the zodiacal dust band and annual variations due to the geometry of the cloud were significant, so simple zodiacal light models could not be used. A more complex model was required, involving an accurate characterization of the solar off-axis contribution to the background. Alternatively, the zodiacal light could be compensated by subtracting a background derived directly from the data. This method included both running differences (subtracting the previous-orbits map), and subtracting a three-day median sky map. A further refinement applied a Gaussian temporal filter to remove most of the long-term (2-week) zodiacal light variation that was not directly removed in the analysis. 


\subsection{Data Processing}

Several techniques were developed to process the SMEI data research groups within the SMEI team, and are summarized below.

3.3.1 The Air Force Research Laboratory (AFRL) Pipeline. This procedure was developed pre-flight but evolved over the first two years of the mission. It was made specifically for the purpose of producing “quick-look” maps for rapid space weather forecasting. Sky maps were produced, correcting for bad pixels, cosmic-ray contamination, detector dark charge, flat-field variations, and attitude jitter. An AFRL zodiacal model was adapted to the SMEI spectral bandpass and a mean stellar background map was created to remove the bright stars and diffuse Galactic emission. The model worked well at removing the large-scale zodiacal light for elongations greater than around $30^{\circ}$ from the Sun, but nearer proved problematic. A secondary mean sky map was calculated using the first three of the four previous orbits (skipping the orbit immediately prior to the observed orbit and neglecting any with significant contamination) and subtracted as a background. The processing pipeline originally took around 90 minutes to import a telemetry file and produce the processed sky maps, but two years into the mission streamlining reduced the processing time to 45 minutes. Additionally, the AFRL pipeline incorporated procedures for mitigating flipper pixels, and removing bright single-frame transient phenomena such as space debris.

3.3.2 The University of California - San Diego (UCSD) Pipeline. This procedure imported the SMEI data into a UCSD database and then performed a conditioning to remove the CCD electronic offset and temperature-dependent dark current [17]. Next, an "indexing" process placed individual frames onto a $0.1^{\circ}$ high-resolution sidereal grid using the known spacecraft pointing information. At this indexing stage, further conditioning removed the bulk of the effects of high-energy particle hits, some of the more fast-moving space debris inside the field of view, and pixels with sudden discrete state changes (flipping pixels). From these sidereal maps, bright stars, background stars, and a zodiacal cloud model were removed.

3.3.3 The National Solar Observatory (NSO) Pipeline. This procedure was developed by the University of Birmingham (UK) and NSO groups [18]. Briefly, once the background contributions from each frame (including CCD electronic offset and dark charge, together with glare) were removed and gain correction applied, known bright artifacts (the Sun, Moon, Venus, etc.), hot pixels and pixels affected by particle hits were identified and flagged. A star removal technique was applied (discussed in the next section), using the same star list as was used in the UCSD pipeline. Further background subtraction was then applied to remove the zodiacal light and light from the ambient solar wind. This final background-removal stage could be performed in several ways, most commonly using a running difference or a three- or six-orbit base subtraction where the median of previous and following orbits was subtracted. 


\subsection{Star Removal}

As mentioned previously, several methods were developed to remove the stars from SMEI data. Two of these removed stars after the formation of single-orbit sky maps, while a third removed them directly from the SMEI image frames.

Attempts were made to reduce the stellar background during the development of the AFRL pipeline, but such reductions were difficult to achieve given SMEI's irregular fish-shaped PSF. A method was devised to "circularize" the PSF by convolving it with its transpose and "stretching” the result until a circularly symmetric PSF of about one degree in size was obtained. This mean PSF was built up from a six-month average, to minimize any residuals from the zodiacal model subtraction. However, this scheme left too many stellar residuals as the circularization preserved much of the high frequency components in the PSF. Also, the background was never fully reduced to the noise level needed for CME detection. Since modeling and removing this stray light would have involved enormous effort, this background method was eventually abandoned.

The UCSD pipeline removed stars from individual sky maps. This involved fitting a standard PSF to known stellar positions for the brightest 6000 stars [19]. The PSF was rotated according to the passage of the SMEI camera sweep over the sky, and according to the location of the star relative to the center of the camera frame. A least-squares fitting procedure adjusted the stellar brightness, a uniform background beneath the PSF, and the location of the stellar centroid position [20].

The NSO pipeline produced an empirical grid of PSFs at an array of locations within an individual frame's FOV, by summing the images around the point when a bright star was centered there; then a feature of that shape was subtracted from the locations of known bright stars (brighter than magnitude 6). Finally, the remaining faint stars and the galactic plane were subtracted using a two-year median map of the sky. This process was effective, but residuals remained for two main reasons: firstly, when stars were close together the fitting of the PSF shape could be somewhat compromised; and secondly, as the mission progressed, there was some excess scattering in the brightest stars making their PSFs somewhat wider than the above grid of PSFs predicted.

\section{RESULTS AND DISCUSSION}

\subsection{Summary of Results}

SMEI routinely imaged almost the entire sky in white light to deeper than 10th magnitude per square degree, producing up to 15 sky maps per day for about 8-1/2 years. This unique dataset provided many opportunities for scientific advancements, not just in solar physics and space weather, but also in auroral physics and in astrophysics, including:

4.1.1 Coronal Mass Ejections. The primary mission of SMEI focused on CMEs. SMEI immediately proved able to detect heliospheric CMEs out to and beyond $90^{\circ}$ elongation, and continued to observe them right up until its last day of operation (a CME was in fact observed on 
the morning of the shutdown). As of early 2015, a total of 391 CMEs have been confirmed throughout the mission. Preliminary catalogs have been published [21, 22] and a complete catalog is available at http://smei.nso.edu/.

The first SMEI results paper [23] reported a CME seen in May 2003. This was the first major geoeffective CME observed during the SMEI mission. The distance-time curve for this event, which was extrapolated backwards to the Sun, matched well in timing with two CMEs that were launched from the Sun early on 28 May and observed by the LASCO coronagraph aboard SOHO. Two interplanetary shocks were measured by the Advanced Composition Explorer (ACE) spacecraft [24] on 29 May, and a major geomagnetic storm began towards the end of the same day. This combination of SMEI sky maps, coronagraph images, and in-situ datasets proved to be a powerful tool, and enabled for the first time almost continuous visual tracking of CMEs from their solar origins through the heliosphere to the Earth.

Towards the end of 2006, the twin STEREO spacecraft [25] were launched carrying, among other instruments, their Heliospheric Imager (HI) ensembles [26]. The HIs provided an excellent dataset to complement SMEI, as their images were free of many of the noise sources in Earth orbit that affected the SMEI data (e.g., magnetospheric energetic particles, hot pixels, Moon), but the HIs are limited in their fields of view. They therefore do not see large parts of the sky to the north and south of the ecliptic. Also, they obtain their images by long exposures, so they produce somewhat "blurred" images of transient phenomena. The first analysis combining the two instruments for a single CME shows that structures with large gradients (i.e., edges) are emphasized in "running-average" maps, whereas sky maps in which a long-temporal baseline is removed show more dense and extensive CME structure trailing the high-gradient features [27].

Other work includes comparison of SMEI CME observations with interplanetary scintillation (IPS) radio data [28] and comparison with in-situ data [29]. One CME that was tracked to 5 AU (i.e., almost to Jupiter) using LASCO, SMEI and Ulysses data [30].

4.1.2 Three-Dimensional (3-D) Reconstruction of CMEs. The angular distances measured by a heliospheric imager cannot be converted to linear distances using the simplifying assumptions that are applied to coronagraph images. At the larger angles from the Sun observed by heliospheric imagers, the "plane of the sky" ceases to be a plane. Instead, the locus of all points closest to the Sun for all lines-of-sight forms a sphere with its diameter as the Sun-observer line, the so-called "Thomson surface" or "Thomson sphere" [31, 32, 33]. At elongations larger than around $45^{\circ}$ it has been shown that a complete 3-D treatment is unavoidable. This is because at large angles the structure of the CME begins to affect the location where the lines-of-sight cross the structure.

The 3-D treatment of the CME location and density distribution has been addressed with three different approaches: (1) empirical reconstruction of the solar wind and transient structure by the conversion of SMEI brightness units into density via tomographic techniques; (2) direct comparison of SMEI maps with synthetic sky maps produced from existing solar wind magnetohydrodynamic (MHD) models; and (3) empirical reconstruction of transients using leading-edge measurements.

(1) Empirical reconstruction of the solar wind extends a technique that originated with Helios data [34] and was subsequently applied to IPS datasets [35]. This technique applies the known physics of the heliosphere and Computer Aided Tomography (CAT) techniques to heliospheric brightness viewed remotely. Briefly, for the time-dependent tomography used in the 
SMEI analysis, the solar wind is reconstructed by projecting it outward from a source surface, assuming radial outflow, and conservation of mass and mass flux to obtain the three-

dimensional solar wind speed and density. SMEI sky maps, where a long-term base is subtracted, and heliospheric in-situ and IPS velocity measurements, are used to improve the solution for the density and velocity of material everywhere along the lines of sight.

The UCSD 3-D time-dependent reconstructions provide CME mass estimates that compared well with coronagraph measurements of individual portions of CMEs [36]. In these analyses, various portions of the CME response were measured (the shock sheath, the erupting prominence material), and compared with equivalent portions of the CME in coronagraph observations. An analysis of the 28 October 2003 CME showed that the prominence material of the CME is elongated in the direction of the Earth and approximates the loop-like magnetic field structure measured in-situ at Earth for this event $[37,38]$. The SMEI reconstructions agree well with insitu density measurements at Earth and STEREO [39].

(2) Model comparison involves processing simulated SMEI sky maps using existing solar wind and interplanetary transient models, for direct comparison with actual SMEI data. The two models that have been used with SMEI for this purpose are HAFv2 [40] and WSA-Enlil+Cone [41]. Both models begin with an MHD solar wind model, constructed using empirical measurements of the lower solar atmosphere. In summary, HAFv2 treats the CME as a kinematic disturbance or pulse in the solar wind medium, determined by solar flare and Type II radio burst data for the boundary conditions. WSA-Enlil+Cone introduces the CME as a conical parcel of plasma, the density, structure and kinematics of which are determined by coronagraph measurements. Both models propagate the created disturbance out to $1 \mathrm{AU}$ and beyond, enabling direct comparison with SMEI data.

(3) Leading-edge comparison is based on the fact that the leading edge of a CME is an easy feature to measure, and investigations show that the location of the apparent leading edge of a CME does not vary significantly with large variations in a simple simulated CME's density [42]. This enables a simplified approach using geometry alone to govern the structure and trajectory of the measured CME. The Tappin-Howard (TH) Model [43] compares a hypercube of simulated leading edges with actual leading edge measurements from SMEI, using a convergence routine involving a genetic algorithm. A basic structure of a Sun-centered spherical arc is assumed, distorted from the perfect sphere with a distortion function. The result is a set of parameters from the simulation that best describes the structure and orientation of the leading edge (latitude and longitude location, and width, distortion and speed), along with uncertainty contours describing the "goodness" of each parameter convergence.

All these 3-D reconstruction results demonstrate that information is available in heliospheric imager data that is unavailable in coronagraph data. With careful analysis, 3-D structural and kinematic information can be extracted directly from heliospheric imager data without the need for auxiliary datasets. The 3-D reconstruction of near-Earth features is a major milestone of the SMEI mission. A number of complementary 3-D reconstruction techniques have subsequently been developed for use with STEREO/HI data, including, for example, the Harmonic-Mean method [44] and forward modeling $[45,46]$.

4.1.3 Corotating Interaction Regions. Corotating interaction regions (CIRs) [47] arise from the interaction between a fast-flowing stream of solar wind material from a coronal hole and the 
surrounding slow solar wind, which causes a compression along the interface that eventually develops into a shock at large distances from the Sun.

Because of their dominance far from the Sun, it was once thought that SMEI would regularly observe CIRs, especially during periods of low solar activity. However, CIR activity was observed only occasionally. The first one was reported [48] using observations in November 2008, near solar minimum. It was identified by its slow relative speed and location at elongations beyond $100^{\circ}$, where CMEs are almost never observed. A second was identified in tomographic reconstructed data, interacting with a CME [49]. CIRs are now detected in white light regularly in STEREO/HI data, with the signature to the east of the Sun-spacecraft line easily identified using elongation-time “J- maps” [50, 51].

4.1.4 Operational Aspects: Space Weather. Although SMEI was a proof-of-concept mission, it was intended not only to demonstrate the ability to detect and track CMEs propagating through the inner heliosphere, together with providing an on-orbit validation of the instrument design with its many novel features, but also to investigate whether heliospheric imaging data could be used to improve the quality of Air Force space weather forecasting.

When SMEI was launched, it was well established that those CMEs most likely to be Earthimpacting are the so-called "halo CMEs". A halo CME has a considerable component directed along the Sun-Earth vector, and, hence, appears in a near-Earth coronagraph such as LASCO as a halo surrounding the Sun $[\mathbf{5 2}, \mathbf{5 3}]$. Such CMEs can be near-sided (i.e., toward the Earth) or farsided (i.e., away from the Earth), and while it is impossible to distinguish the two solely with coronagraphic measurements, this is possible using heliospheric imager data. Furthermore, with the exception of IPS radio measurements and the old data from Helios, the large gap in space between the outer edge of a coronagraph's FOV and near-Earth in-situ measurement was inaccessible prior to the advent of SMEI. Predictions of CME arrival time, speed, and geoeffectiveness were therefore limited to models that extrapolated the evolution of the CME using data from coronagraphs viewing close to the Sun, or short timescale ( $\sim 1$ hour or less) alerts from in-situ measurements. SMEI bridged the gap between the Sun and the Earth, and enabled comparison with forecast models. Even so, because one could typically not identify and track a CME reliably until it was closer than 0.5 AU to the Earth, predictions often became available only a day or so before the CME actually arrived at Earth [54].

The first attempts to use SMEI for space weather forecasting associated post-facto interplanetary shock measurements near the Earth with LASCO halo CMEs by projecting SMEI distance-time plots forward to the shock and backward to the halo CME [55, 56]. By forwardprojecting the SMEI distance-time plot, an estimate of the CME's arrival time at 1 AU could be made, which was compared with the in-situ shock measurements.

AFRL considered the demonstration of the capability of forecasting as a primary goal of the SMEI mission. As stated previously, the explicit goal of the AFRL processing pipeline was the production of a computationally-rapid sky map construction system for near-real-time space weather forecasting. To this end, they created a processing pipeline that met the AFRL requirement to deliver SMEI products (including maps) within six hours of the original data having been obtained on the spacecraft. In practice, SMEI had too high a data latency to actually do such forecasting (although it was successful for a few events). 
Eventually, the 3-D reconstruction techniques discussed previously were all applied to the space weather forecasting effort. In particular, the Tappin-Howard Model (“T-H Model”) [57, 58] enabled a speedy evaluation of CME leading edges and issued arrival time and speed forecasts as each new SMEI sky map was formed. Using this technique, an RMS accuracy of 10 hours, compared to the previous best case of 11 hours, was achieved [59], and an accuracy as good as two hours was achieved for some cases [60, 61]. SMEI therefore demonstrated that heliospheric imager data can be a useful addition to the space weather forecasting toolbox.

4.1.5 Operational Aspects: Resident Space Objects and Orbital Debris. The panoramic imaging capability of SMEI resulted in the detection of a large number of Earth-orbiting objects (resident space objects - RSOs). SMEI observed RSOs at a rate of about one per minute. Sometimes, they were far enough away that they drifted through the SMEI FOV against the backdrop of stars over several sequential four-second exposures, occasionally being observed for up to 10 minutes when orbital geometries were favorable. However, the majority of RSOs appeared as streaks that crossed the entire in-scan field in a single exposure. Roughly a third of all the extracted RSOs were not associated with objects whose positions were predicted using the publicly available two-line orbital element sets [62].

SMEI occasionally observed a "swarm" of objects that was manifest as a sudden, relatively brief occurrence of a large number of RSOs, many more than the normal rate, up to dozens detected in a four-second exposure [63]. These swarms usually lasted for a few minutes and generally crossed the field of view in a consistent direction. The likely explanation is that the swarms were created by space debris striking Coriolis and ejecting a large number of smaller objects, most likely from the spacecraft multi-layer insulation (MLI). Similar particle swarm events are fairly frequently observed with the SOHO coronagraphs and the STEREO HIs, and are attributed to interplanetary dust or micrometeorite particles striking the spacecraft MLI and causing the release of many smaller pieces of debris.

4.1.6 High-Altitude Aurora. While aurorae have been observed at altitudes reaching $1000 \mathrm{~km}$ [64], these are rare. Since SMEI viewed only above its $842 \mathrm{~km}$ altitude, it was initially assumed that aurorae would be seldom observed. This assumption proved to be false, with aurorae often saturating the SMEI cameras, most significantly during times of enhanced geomagnetic activity (i.e., when $\mathrm{Kp}>4$ ).

A survey of aurorae observed by SMEI during its first year operation [65] divided the auroral signatures into two categories: (1) a "flash" having bright regions where all three cameras were briefly illuminated simultaneously for 1-3 minutes); and (2) a "streamer" with one or more filaments curving asymptotically with increasing time toward the rear of the satellite. Both are diffuse features, easily distinguished from particle strikes that affect only one or a few pixels on a given CCD.

During SMEIs first year, a total of 823 SMEI orbits with flashes and 219 with streamers were observed: an average of around one auroral signature every five days. A good correlation with geomagnetic activity indices was found. Flashes occurred as the spacecraft passed directly through a region brightly illuminated by the aurora, and the streamers as SMEI viewed columns of luminous material some distance away from the spacecraft. 
4.1.7 Comets. SMEI's wide FOV enabled comet-tail observations that were previously unfeasible. SMEI provided the first observations of comet tail disconnection events at very large distances from the comet's nucleus $[\mathbf{6 6 , 6 7 , 6 8}$, including six tail disruptions for comets NEAT and LINEAR. One such disruption was noteworthy as it corresponded to the passage of a faint $\mathrm{CME}$ at the time of disruption. This shows that at least one disconnection event was likely caused by a CME, but the cause of the remaining five was uncertain: one suggested possibility was a heliospheric current-sheet crossing. The disconnection first appeared as a kink moving anti-sunward that eventually developed knots within the tail. The disconnected part became a circular arc roughly normal to the solar radial vector, which then moved away from the Sun. Similar comet disconnection events were subsequently observed using STEREO/HI [69, 70]. An outburst of Comet 17P/Holmes using SMEI data has also been reported recently [71].

In addition to complete disconnections, SMEI demonstrated that comet tails are sensitive indicators of variations in the solar wind [72], as shown by comparing the radial speeds of smallscale tail disruptions observed by SMEI as comets move away from the Sun with in-situ and IPS solar wind speed measurements near the Earth. The poor correlation indicates disparities between the sizes of the disruptions measured by the auxiliary datasets compared with those observed by SMEI.

This analysis of comet-tail motions shows that the solar wind outflow is chaotic and highly variable, and not the benign regular outward motion of a quiescent plasma. Such comet-tail observations, using heliospheric white-light imagers, are important because they can provide solar wind information closer to the Sun than where spacecraft, with their in-situ measurements, have ventured to date.

4.1.8 Stellar Astrophysics. Stars brighter than 6th magnitude observed by SMEI were individually fitted and removed from the SMEI data for the CME analyses. However, the time series derived as a byproduct of this processing have also proven to be a valuable resource for stellar variability studies in their own right.

Using SMEI observations in conjunction with other ground and space data, studies of Polaris ( $\alpha$ Ursae Minoris), a well-known Cepheid variable, revealed an increase in amplitude of oscillation by around $30 \%$ over $4-1 / 2$ years, reversing a decrease in the amplitude observed previously [73, 74]. This finding challenges claims that Polaris is moving towards the so-called instability strip. Other work used SMEI to study the eclipsing binary star $\psi$ Centauri [75], oscillations in $\beta$ Ursae Minoris and $\gamma$ Doradus [76, 77], and astereoseismology of red giant stars, including Arcturus ( $\alpha$ Boötis) and $\beta$ Ursae Minoris [78].

SMEI also provided a substantial increase in data for novae, particularly on the rising phase, which previously was poorly characterized [79]. Light curves with unprecedented temporal resolution from the rise phase of four novae enabled accurate identification of the epoch of initial explosion, as well as investigation of the duration of pre-maximum halt, the presence or absence of secondary maxima, and the speed of decline of the initial light curve. For one event, the precise timing of the onset of dust formation was identified, which led to an estimation of the bolometric luminosity, white dwarf mass, and object distance. This work emphasizes the fact that 
many bright novae, particularly those with short duration outbursts, are currently overlooked or are observed after the rise, considering that most instruments do not view the sky all the time.

A search for optical signatures accompanying gamma-ray bursts yielded no detections at the sensitivity limit of SMEI [80].

4.1.9 Zodiacal Light and the Gegenschein. Previously in this Report, the zodiacal light was presented as a nuisance that had to be removed for the study of CMEs. However, after star removal and a carefully designed subtraction of instrumental background, it is also possible to study the background scattered sunlight, the zodiacal light, from measurements by SMEI, and photometric R-band measurements of the zodiacal dust bands have been reported [81, 82].

A portion of the zodiacal light, the Gegenschein, is a diffuse brightening of the night sky centered on the anti-solar point in the sky. It is caused by sunlight back-scattered from dust in the interplanetary medium, and is sometimes distinguished from the rest of the zodiacal light because the high angle of reflection of the sunlight allows a more efficient scattering process. An enigmatic issue involves the detailed distribution of the scattering dust relative to the Earth, which requires an accurate characterization combined with a scattering phase function. SMEI data have been used to characterize the Gegenschein [83]. Formulae were developed to model the zodiacal light surface brightness in the anti-solar hemisphere and then applied to describe the brightness distribution, shape, and variation with time, confirming the commonly-held view that the brightness distribution has a peak at the anti-solar point that rises above the broader background.

\subsection{Considerations and Concepts for Future Heliospheric Imagers}

Heliospheric imagers very similar to the STEREO/HIs are currently being developed for the next generation of solar spacecraft observatories (SoloHI for Solar Orbiter [84] and WISPR for Solar Probe Plus [85]) that are scheduled for launch near the end of the decade. In addition, other more general concepts and guidelines have been proposed for possible future missions [86].

Any future heliospheric imager should consider the lessons learned from SMEI and the STEREO/HIs to develop a better quality imager and enable more accurate calibration and scientific data extractions [87]. To this end, Buffington et al. (1998); Jackson et al. (2010a, 2011a) have proposed the All-Sky Imager (ASI) has been proposed [88, 89]. This instrument views almost an entire hemisphere of directions relative to the Sun, from $2^{\circ}$ out as far as $180^{\circ}$. The instrument uses a mirror-lens optical system in order to provide a very large FOV while minimizing scattering of light from bright objects, and would be suitable for deployment far enough away from low-Earth orbit that interference from the Moon would not be a problem.

Recent theoretical work [90] has demonstrated that additional information and kinematic parameter and density calculation precision could be obtained if heliospheric imagers had the capability to observe in polarized as well as unpolarized light, and a concept for a polarizing heliospheric imager has been proposed [91]. 
A special configuration of space weather interest occurred in 2009-2010 when STEREO- B was in the vicinity of the L5 Lagrange point, which is located $60^{\circ}$ east of the Sun-Earth line. Observations at or near this location permitted the use of STEREO-B as a pathfinder for a future space weather monitoring mission at L5 that could include heliospheric imaging capabilities [92]. In particular, the speed and size of an Earth-directed CME is perhaps more easily determined from a side view than a head-on view, and that the side view might enable the observation of such a CME at an earlier time than is feasible with the head-on view. In addition, from this location, active regions and coronal holes can be viewed before they arrive on the Earth-facing disk, and their location, size and activity assessed. Finally, the geoeffective space weather resulting from high speed solar wind streams, such as radiation belt relativistic electron enhancements, can be forecast days in advance from L5. Offsetting these advantages would be the cost and telemetry requirements for deploying at and communicating with this distant location. The Earth-Affecting Solar Causes Observatory (EASCO) [93] is one proposed mission to be located at the Sun-Earth L5.

\section{CONCLUSIONS}

SMEI was the first of a new class of solar and space imager. This Technical Report has reviewed the many contributions of SMEI to space science, space weather, and astrophysics. As a proof-of-concept mission on a limited budget, SMEI was highly successful. SMEI achieved its primary objective during the first month of mission operations, and went on to provide many further years of unique data. As a prototype for a new class of imager, SMEI served as an invaluable pathfinder, and leaves an important legacy. SMEI proved not only that CMEs can be observed, tracked, and quantitatively measured during their passage throughout the inner heliosphere, but also that information about CMEs can be extracted from heliospheric imagers that is unavailable from coronagraphs. 


\section{REFERENCES}

[1] Eyles, C. J., Simnett, G. M., Cooke, M. P., Jackson, B. V., Buffington, A., Hick, P. P., Waltham, N. R., King, J. M., Anderson, P. A., and Holladay, P. E., “The Solar Mass Ejection Imager (SMEI),” Solar Phys., 217, Nov 2003, pp. 319-347.

[2] Jackson, B. V., Buffington, A., Hick, P. P., Altrock, R. C., Figueroa, S., Holladay, P. E., Johnston, J. C., Kahler, S. W., Mozer, J. B., Price, S., Radick, R. R., Sagalyn, R., Sinclair, D., Simnett, G. M., Eyles, C. J., Cooke, M. P., Tappin, S. J., Kuchar, T., Mizuno, D. R., Webb, D. F., Anderson, P. A., Keil, S. L., Gold, R. E., and Waltham, N. R., “The Solar Mass-Ejection Imager (SMEI) Mission,” Solar Phys., 225, Nov 2004, pp. 177-207.

[3] Kaiser, M. L., Kucera, T. A., Davila, J. M., St. Cyr, O. C., Guhathakurta, M., and Christian, E., “The STEREO Mission: An Introduction,” Space Sci. Rev., 136, Apr 2008, pp. 5-16.

[4] Eyles, C. J., Harrison, R. A., Davis, C. J., Waltham, N. R., Shaughnessy, B. M., MapsonMenard, H. C. A., Bewsher, D., Crothers, S. R., Davies, J. A., Simnett, G. M., Howard, R. A., Moses, J. D., Newmark, J. S., Socker, D. G., Halain, J.-P., Defise, J.-M., Mazy, E., and Rochus, P., “The Heliospheric Imagers Onboard the STEREO Mission,” Solar Phys., 254, Jan 2009, pp. 387-445.

[5] Leinert, C., Link, H., Pitz, E., Salm, N., and Knueppelberg, D., “The Helios Zodiacal Light Experiment /E 9/,” Raumfahrtforschung, 19, Oct 1975, pp. 264-267.

[6] Jackson, B.V., "Imaging of Coronal Mass Ejections by the HELIOS Spacecraft," Solar Phys., 100, Oct 1985, pp. 563-574.

[7] Webb, D. F., Mizuno, D. R., Buffington, A., Cooke, M. P., Eyles, C. J., Fry, C. D., Gentile, L. C., Hick, P. P., Holladay, P. E., Howard, T. A., Hewitt, J. G., Jackson, B. V., Johnston, J. C., Kuchar, T. A., Mozer, J. B., Price, S., Radick, R. R., Simnett, G. M., and Tappin, S. J., "Solar Mass Ejection Imager (SMEI) Observations of Coronal Mass Ejections (CMEs) in the Heliosphere,” J. Geophys. Res., 111, A12101, Dec 2006, doi:10.1029/2006JA011655.

[8] Howard, Timothy, Coronal Mass Ejections: An Introduction, Springer Astrophysics and Space Science Library, Vol. 376, New York, NY, 2011, pp. 1-244.

[9] Howard, Timothy, Space Weather and Coronal Mass Ejections, Springer Briefs in Astronomy, New York, NY, 2014, pp. 1-107.

[10] Cliver, E. W. and Dietrich, W. F., “The 1859 Space Weather Event Revisited: Limits of Extreme Activity,” J. Space Weather and Space Clim., 3, Oct 2013, pp. 1-15.

[11] Tousey, R., “The Solar Corona,” Space Research XIII, 2, 1973, pp.713-730. 
[12] Brueckner, G. E., Howard, R. A., Koomen, M. J., Korendyke, C. M., Michels, D. J., Moses, J. D., Socker, D. G., Dere, K. P., Lamy, P. L., Llebaria, A., Bout, M. V., Schwenn, R., Simnett, G. M., Bedford, D. K., and Eyles, C. J., "The Large Angle Spectroscopic Coronagraph (LASCO),” Solar Phys., 162, Dec 1995, pp. 357-402.

[13] Howard, T. A., Bisi, M. M., Buffington, A, Clover, J. M., Cooke, M. P., Eyles, C. J., Hick, P. P., Holladay, P. E., Jackson, B. V., Johnston, J. C., Kahler, S. W., Kuchar, T. A., Mizuno, D. R., Penny, A. J., Price, S. D., Radick, R. R., Simnett, G. M., Tappin, S. J., Waltham, N. R., and Webb, D. F., “The Solar Mass Ejection Imager and Its Heliospheric Imaging Legacy,” Space Sci. Rev., 180, Dec 2013, pp. 1-38.

[14] Gaiser, P. W., St. Germain, K. M., Twarog, E. M., Poe, G. A., Purdy, W., Richardson, D., Grossman, W., Jones, W. L., Spencer, D., Golba, G., Cleveland, J., Choy, L, Bevilacqua, R. M., and Chang, P. S., "The WindSat Spaceborne Polarimetric Microwave Radiometer: Sensor Description and Early Orbit Performance,” IEEE Trans. on Geosci. and Remote Sensing, 42, Nov 2004, pp. 2347-2361.

[15] Eyles, C. J., Simnett, G. M., Cooke, M. P., Jackson, B. V., Buffington, A., Hick, P. P., Waltham, N. R., King, J. M., Anderson, P. A., and Holladay, P. E., "The Solar Mass Ejection Imager (SMEI),” Solar Phys., 217, Nov 2003, pp. 319-347.

[16] Buffington, A., Jackson, B. V., and Hick, P. P., "Calculations for and Laboratory Measurements of a Multistage Labyrinthine Baffle for SMEI,” Proc. SPIE, 4853, 2003, pp. 490-503.

[17] Hick, P. P., Buffington, A., and Jackson, B. V., “The SMEI Real-Time Data Pipeline: From Raw CCD Frames to Photometrically Accurate Full-Sky Maps,” Proc. SPIE, 5901, Aug 2005, pp. 340-346.

[18] Tappin, S. J., Howard, T. A., Hampson, M. M., Thompson, R. N., and Burns, C. E., “On the Autonomous Detection of Coronal Mass Ejections in Heliospheric Imager Data,” J. Geophys. Res., 117, A05103, May 2012, doi:1029/2011JA017439.

[19] Hick, P. P., Buffington, A., and Jackson, B. V., "A Procedure for Fitting Point Sources in SMEI White-Light Full-Sky Maps,” Proc. SPIE, 6689, Oct 2007, doi:10.1117/12.734808.

[20] Hounsell, R., Bode, M. F., Hick, P. P., Buffington, A., Jackson, B. V., Clover, J. M., Shafter, A. W., Darnley, M. J., Mawson, N. R., Steele, I. A., Evans, A., Eyres, S. P. S., and O’Brien, T. J., "Exquisite Nova Light Curves from the Solar Mass Ejection Imager (SMEI)," Astrophys. J., 724, Nov 2010, pp. 480-486. 
[21] Webb, D. F., Mizuno, D. R., Buffington, A., Cooke, M. P., Eyles, C. J., Fry, C. D., Gentile, L. C., Hick, P. P., Holladay, P. E., Howard, T. A., Hewitt, J. G., Jackson, B. V., Johnston, J. C., Kuchar, T. A., Mozer, J. B., Price, S., Radick, R. R., Simnett, G. M., and Tappin, S. J., "Solar Mass Ejection Imager (SMEI) Observations of Coronal Mass Ejections (CMEs) in the Heliosphere,” J. Geophys. Res., 111, A12101, Dec 2006, doi:10.1029/2006JA011655.

[22] Howard, T. A. and Simnett, G. M., "Interplanetary Coronal Mass Ejections That Are Undetected by Solar Coronagraphs,” J. Geophys. Res., 113, A08102, Aug 2008, doi:10.1029/2007JA012920.

[23] Tappin, S. J., Buffington, A., Cooke, M. P., Eyles, C. J., Hick, P. P., Holladay, P. E., Jackson, B. V., Johnston, J. C., Kuchar, T., Mizuno, D., Mozer, J. B., Price, S., Radick, R. R., Simnett, G. M., Sinclair, D., Waltham, N. R., and Webb, D. F., “Tracking a Major Interplanetary Disturbance with SMEI,” Geophys. Res. Lett., 31, L02802, Jan 2004, doi:10.1029/2003GL018766.

[24] Stone, E. C., Frandsen, A. M., Mewaldt, R. A., Christian E. R., Margolies, D., Ormes J. F., and Snow F., “The Advanced Composition Explorer,” Space Science Rev., 86, Jul 1998, pp. 1-22.

[25] Kaiser, M. L., Kucera, T. A., Davila, J. M., St. Cyr, O. C., Guhathakurta, M., and Christian, E., “The STEREO Mission: An Introduction,” Space Sci. Rev., 136, Apr 2008, pp. 5-16.

[26] Eyles, C. J., Harrison, R. A., Davis, C. J., Waltham, N. R., Shaughnessy, B. M., MapsonMenard, H. C. A., Bewsher, D., Crothers, S. R., Davies, J. A., Simnett, G. M., Howard, R. A., Moses, J. D., Newmark, J. S., Socker, D. G., Halain, J.-P., Defise, J.-M., Mazy, E., and Rochus, P., “The Heliospheric Imagers Onboard the STEREO Mission,” Solar Phys., 254, Jan 2009, pp. 387-445.

[27] Webb, D. F., Howard, T. A., Fry, C. D., Kuchar, T. A., Odstrĉil, D., Jackson, B. V., Bisi, M. M., Harrison, R. A., Morrill, J. S., Howard, R. A., and Johnston, J. C., "Study of CME Propagation in the Inner Heliosphere: SOHO LASCO, SMEI, and STEREO HI Observations of the January 2007 Events,” Solar Phys., 256, May 2009, pp. 239-267.

[28] Jackson, B. V., Hick, P. P., Buffington, A., Bisi, M. M., Clover, J. M., Tokumaru, M., Kojima, M., and Fujiki, K., J., "Solar Mass Ejection Imager (SMEI) 3-D Reconstruction of Density Enhancements Behind Interplanetary Shocks: In-situ Comparison Near Earth and at STEREO,” Atmos. Solar-Terr. Phys., 73, Jul 2011, pp. 1214-1227.

[29] Webb, D. F., Mostl, C., Jackson, B. V., Bisi, M. M., Howard, T. A., Mulligan, T., Jensen, E. A., Jian, L. K., Davies, J. A., de Koning, C. A., Liu, Y., Temmer, M., Clover, J. M., Farrugia, C. J., Harrison, R. A., Nitta, N., Odstrĉil, D., Tappin, S. J., and Yu, H.-S., "Heliospheric Imaging of 3D Density Structures During the Multiple Coronal Mass Ejections of Late July to Early August 2010,” Solar Phys., 285, Jul 2013, pp. 317-348. 
[30] Tappin, S. J., "The Deceleration of an Interplanetary Transient from the Sun to 5 AU," Solar Phys., 233, Feb 2006, pp. 233-248.

[31] Vourlidas, A. and Howard, R. A., “The Proper Treatment of Coronal Mass Ejection Brightness: A New Methodology and Implications for Observations,” Astrophys. J., 642, May 2006, pp. 1216-1221.

[32] Howard, T. A. and Tappin, S. J., "Interplanetary Coronal Mass Ejections Observed in the Heliosphere: 1. Review of Theory,” Space Sci. Rev., 147, Oct 2009, pp. 31-54.

[33] Howard, T. A. and DeForest, C. E., "Inner Heliosphere Flux Rope Evolution via Imaging of Coronal Mass Ejections,” Astrophys. J., 746, Feb 2012, pp. 64-75.

[34] Jackson, B. V., and Leinert, C., J., “HELIOS Images of Solar Mass Ejections,” Geophys. Res., 90, Nov 1985, pp. 10759-10764.

[35] Jackson, B. V., Hick, P. P., Buffington, A., Bisi, M. M., Clover, J. M., Tokumaru, M., Kojima, M., and Fujiki, K., J., “Three-Dimensional Reconstruction of Heliospheric Structure Using Iterative Tomography: A Review,” Atmos. Solar-Terr. Phys., 73, Jun 2011, pp. 1214-1227.

[36] Jackson, B. V., Bisi, M. M., Hick, P. P., Buffington, A., Clover, J. M., and Sun, W., J., "Solar Mass Ejection Imager 3-D Reconstruction of the 27-28 May 2003 Coronal Mass Ejection Sequence,” Geophys. Res., 113, A00A15, Mar 2008, doi:10.1029/2008JA013224.

[37] Jackson, B. V., Buffington, A., Hick, P. P., Wang, X., and Webb, D., "Preliminary Three-Dimensional Analysis of the Heliospheric Response to the 28 October 2993 CME Using SMEI White-Light Observations,” J. Geophys. Res., 111, A04S91, Apr 2006, doi:10.1029/2004JA010942.

[38] Jensen, E. A., Hick, P. P., Bisi, M. M., Jackson, B. V., Clover, J., and Mulligan, T., "Faraday Rotation Response to Coronal Mass Ejection Structure,” Solar Phys., 265, Aug 2010, pp. 31-48.

[39] Jackson, B. V., Hick, P. P., Buffington, A., Clover, J. M., and Tokumaru, M., "Forecasting Transient Heliospheric Solar Wind Parameters at the Locations of the Inner Planets,” Adv. Geosciences, 30, Oct 2012, pp. 93-115.

[40] Fry, C. D., Sun, W., Deehr, C. S., Dryer, M., Smith, Z., Akasofu, S.-I., Tokumaru, M., and Kojima, M., "Improvements to the HAF Solar Wind Model for Space Weather Predictions," J. Geophys. Res., 106, Oct 2001, pp. 20985-21002.

[41] Odstrĉil, D., Riley, P., and Zhao, X. P., “Numerical Simulation of the 12 May 1997 Interplanetary CME Event,” J. Geophys. Res., 109, A02116, Feb 2004, doi:10.1029/2003JA010135. 
[42] Howard, T. A., and Tappin, S. J., "Recreating the Three Dimensional Structure of Interplanetary Coronal Mass Ejections,” EOS Trans. AGU, 89, Dec 2008, p. SH13B-1531, (abstract).

[43] Tappin, S. J. and Howard, T. A., "Interplanetary Coronal Mass Ejections Observed in the Heliosphere: 2. Model and Data Comparison,” Space Sci. Rev., 147, Oct 2009, pp. 55-87.

[44] Lugaz, N., Vourlidas, A., and Roussev, I. I., "Deriving the Radial Distances of Wide Coronal Mass Ejections from Elongation Measurements in the Heliosphere - Application to CME-CME Interaction,” Ann. Geophys., 27, Sep 2009, pp. 3479-3488.

[45] Xiong, Ming, Breen, A. R., Bisi, M. M., Owens, M. J., Fallows, R. A., Dorrian, G. D., Davies, J. A., and Thomasson, P., "Forward Modeling to Determine the Observational Signatures of White-Light Imaging and Interplanetary Scintillation for the Propagation of an Interplanetary Shock in the Ecliptic Plane,” J. Atmos. Solar Terr. Phys., 73, Jun 2011, pp. 1270-1280.

[46] Xiong, M., Davies, J. A., Bisi, M. M., Owens, M. J., Fallows, R. A., Dorrian, G. D., "Effects of Thomson-Scattering Geometry on White-Light Imaging of an Interplanetary Shock: Synthetic Observations from Forward Magnetohydrodynamic Modeling," Solar Phys., 285, Jul 2013, pp. 369-389.

[47] Smith, E. J., and Wolfe, J. H., "Observations of Interaction Regions and Corotating Shocks Between One and Five AU: Pioneers 10 and 11," Geophys. Res. Lett., 2, 1976, pp. 137-140.

[48] Tappin, S. J. and Howard, T. A., "Direct Observation of a Corotating Interaction Region by Three Spacecraft,” Astrophys. J., 702, Sep 2009, pp. 862-870.

[49] Jackson, B. V., Buffington, A., Hick, P. P., Clover, J. M., Bisi, M. M., and Webb, D. F., "SMEI 3D Reconstruction of a Coronal Mass Ejection Interacting with a Corotating Solar Wind Density Enhancement: The 2008 April 26 CME,” Astrophys. J., 724, Dec 2010, pp. 829-834.

[50] Sheeley, N. R., Jr., Herbst, A. D., Palatchi, C. A., Wang, Y.-M., Howard, R. A., Moses, J. D., Vourlidas, A., Newmark, J. S., Socker, D. G., Plunkett, S. P., Korendyke, C. M., Burlaga, L. F., Davila, J. M., Thompson, W. T., St Cyr, O. C., Harrison, R. A., Davis, C. J., Eyles, C. J., Halain, J. P., Wang, D., Rich, N. B., Battams, K., Esfandiari, E., and Stenborg, G., "SECCHI Observations of the Sun’s Garden-Hose Density Spiral,” Astrophys. J., 674, Feb 2008, pp. L109-L112.

[51] Howard, T. A., DeForest, C. E., and Reinard, A. A., "White-Light Observations of Solar Wind Transients and Comparison with Auxiliary Data Sets,” Astrophys. J., 754, Aug 2012, pp. 102-111.

[52] Howard, R. A., Michels, D. J., Sheeley, N. R., Jr., and Koomen, M. J., “The Observation of a Coronal Transient Directed at Earth,” Astrophys. J., 263, Dec 1982, pp. L101-L104. 
[53] Howard, R. A., Sheeley, N. R., Jr., Koomen, M. J., and Michels, D. J., “Coronal Mass Ejections - 1979-1981,” J. Geophys. Res., 90, Sep 1985, pp. 8173-8191.

[54] Webb, D. F., Howard, T. A., Fry, C. D., Kuchar, T. A., Mizuno, D. R., Johnston, J. C., and Jackson, B. V., "Studying Geoeffective Interplanetary Coronal Mass Ejections Between the Sun and Earth: Space Weather Implications of Solar Mass Ejection Imager Observations,” Space Weather, 7, S05002, May 2009, doi:10.1029/2008SW000409.

[55] Howard, T. A., Simnett, G. M., Robinson, I. M., and Tappin, J., "Earthbound and Geoeffective CMEs Observed by the Solar Mass Ejection Imager,” EOS Trans. AGU, 85, Dec 2004, p. SH11A-04 (abstract).

[56] Howard, T. A., Webb, D. F., Tappin, S. J., Mizuno, D. R., and Johnston, J. C., “Tracking Halo Coronal Mass Ejections from 0.1 AU and Space Weather Forecasting Using the Solar Mass Ejection Imager (SMEI),” J. Geophys. Res., 111, A04105, Apr 2006, doi:10.1029/2005JA011349.

[57] Tappin, S. J. and Howard, T. A., "Interplanetary Coronal Mass Ejections Observed in the Heliosphere: 2. Model and Data Comparison,” Space Sci. Rev., 147, Oct 2009, pp. 55-87.

[58] Howard, T. A. and Tappin, S. J., “Application of a New Phenomenological Coronal Mass Ejection Model to Space Weather Forecasting,” Space Weather, 7, S07004, Jul 2010, doi:10.1029/2009SW000531.

[59] Webb, D. F., Howard, T. A., Fry, C. D., Kuchar, T. A., Mizuno, D. R., Johnston, J. C., and Jackson, B. V., "Studying Geoeffective Interplanetary Coronal Mass Ejections Between the Sun and Earth: Space Weather Implications of Solar Mass Ejection Imager Observations,” Space Weather, 7, S05002, May 2009, doi:10.1029/2008SW000409.

[60] Howard, T. A. and Tappin, S. J., “Application of a New Phenomenological Coronal Mass Ejection Model to Space Weather Forecasting,” Space Weather, 7, S07004, Jul 2010, doi:10.1029/2009SW000531.

[61] Webb, D. F., Johnston, J. C., Kuchar, T. A., Tappin, J., and Howard, T. A., "Forecasting Earth Arrivals of CMEs with Heliospheric Imagers (Invited),” EOS Trans. AGU, 91, Dec 2010, p. SH54D-05 (abstract).

[62] Mizuno, D. R., Price, S. D., Kraemer, K. E., Kuchar, T. A., and Johnston, J. C., “Debris Swarms Seen by SMEI,” Adv. Space Res., 49, Jan 2012, pp. 162-176.

[63] Mizuno, D. R., Price, S. D., Kraemer, K. E., Kuchar, T. A., and Johnston, J. C., “Debris Swarms Seen by SMEI,” Adv. Space Res., 49, Jan 2012, pp. 162-176.

[64] Størmer, C., The Polar Aurora, Clarendon, Oxford, UK, 1955, pp. 1-403. 
[65] Mizuno, D. R., Buffington, A., Cooke, M. P., Eyles, C. J., Hick, P. P., Holladay, P. E., Jackson, B. V., Johnston, J. C., Kuchar, T. A., Mozer, J. B., Price, S. D., Radick, R. R., Simnett, G. M., Sinclair, D., Tappin, S. J., and Webb, D. F., "Very High Altitude Aurora Observations with the Solar Mass Ejection Imager,” J. Geophys. Res., 110, A07230, Jul 2005, doi:10.1029/2004JA010689.

[66] Kuchar, T. A., Mizuno, D., Arge, C. N., Webb, D. F., Kahler, S. W., and Johnston, J. C., “Comet Tail Disconnections Observed by SMEI," EOS Trans. AGU, 85, Dec 2004, p. SH21A0396 (abstract).

[67] Kuchar, T. A., Buffington, A., Howard, T., Arge, C. N., Webb, D. F., Jackson, B. V., and Hick, P. P., "The Evolution of Comets in the Heliosphere as Observed by SMEI," EOS Trans. $A G U, 87$, Dec 2006, p. SH32A-08 (abstract).

[68] Kuchar, T. A., Buffington, A., Arge, C. N., Hick, P. P., Howard, T. A., Jackson, B. V., Johnston, J. C., Mizuno, D. R., Tappin, S. J., and Webb, D. F., "Observations of a Comet Tail Disruption Induced by the Passage of a CME,” J. Geophys. Res., 113, A04101, Apr 2008, doi:10.1029/2007JA012603.

[69] Vourlidas, A., Davis, C. J., Eyles, C. J., Crothers, S. R., Harrison, R. A., Howard, R. A., Moses, J. D., and Socker, D. G., "First Direct Observation of the Interaction between a Comet and a Coronal Mass Ejection Leading to a Complete Plasma Tail Disconnection,” Astrophys. J., 668, Oct 2007, pp. L79-L82.

[70] Clover, J. M., Jackson, B. V., Buffington, A., Hick, P. P., and Bisi, M. M., "Solar Wind Speed Inferred from Cometary Plasma Tails Using Observations from STEREO HI-1,” Astrophys. J., 713, Apr 2010, pp. 394-397.

[71] Li, J., Jewitt, D., Clover, J. M., and Jackson, B. V., “Outburst of Comet 17P/Holmes Observed with the Solar Mass Ejection Imager,” Astrophys. J., 728, Feb 2011, pp. 31-39.

[72] Buffington, A., Bisi, M. M., Clover, J. M., Hick, P. P., Jackson, B. V., and Kuchar, T. A., “Analysis of Plasma Tail Motions for Comets C/2001 Q4 (NEAT) and C/2002 T7 (LINEAR) Using Observations from SMEI,” Astrophys. J., 677, Apr 2008, pp. 798-807.

[73] Penny, A. J., "Variable Stars with the SMEI Instrument," EOS Trans. AGU, 87, Dec 2006, p. SH32A-07 (abstract).

[74] Bruntt, H., Evans, N. R., Stello, D., Penny, A. J., Eaton, J. A., Buzasi, D. L., Sasselov, D. D., Preston, H. L., and Miller-Ricci, E., "Polaris the Cepheid Returns: 4.5 Years of Monitoring from Ground and Space,” Astrophys. J., 683, Aug 2008, pp. 433-440.

[75] Bruntt, H., Southworth, J., Torres, G., Penny, A. J., Clausen, J. V., and Buzasi, D. L., "VizieR Online Data Catalog: $\psi$ Cen Light Curves," Astron. Astrophys., 456, Sep 2006, pp. 651-658. 
[76] Tarrant, N. J., Chaplin, W. J., Elsworth, Y., Spreckley, S. A., and Stevens, I. R., "Oscillations in $\beta$ Ursae Minoris. Observations with SMEI," Astron. Astrophys., 483, Jun 2008, pp. L43-L46.

[77] Tarrant, N. J., Chaplin, W. J., Elsworth, Y., Spreckley, S. A., and Stevens, I. R., “SMEI Observations of Previously Unseen Pulsation Frequencies in $\gamma$ Doradus," Astron. Astrophys., 492, Dec 2008, pp. 167-169.

[78] Tarrant, N. J., Chaplin, W. J., Elsworth, Y. P., Spreckley, S. A., and Stevens, I. R., “Asteroseimology of Red Giant Stars,” Comm. Astereoseismology, 157, Dec 2008, pp. 92-97.

[79] Hounsell, R., Bode, M. F., Hick, P. P., Buffington, A., Jackson, B. V., Clover, J. M., Shafter, A. W., Darnley, M. J., Mawson, N. R., Steele, I. A., Evans, A., Eyres, S. P. S., and O’Brien, T. J., "Exquisite Nova Light Curves from the Solar Mass Ejection Imager (SMEI)," Astrophys. J., 724, Nov 2010, pp. 480-486.

[80] Buffington, A., Band, D. L., Jackson, B. V., Hick, P. P., and Smith, A. C., “A Search for Early Optical Emission at Gamma-Ray Burst Locations by the Solar Mass Ejection Imager (SMEI),” Astrophys. J., 637, Feb 2006, pp. 880-888.

[81] Penny, A. J., "Variable Stars with the SMEI Instrument,” EOS Trans. AGU, 87, Dec 2006, p. SH32A-07 (abstract).

[82] Buffington, A., Jackson, B. V., Hick, P., and Price, S. D., “An Empirical Description of Zodiacal Light as Measured by SMEI,” EOS Trans. AGU, 87, Dec 2006, p. SH11A-04 (abstract).

[83] Buffington, A., Bisi, M. M., Clover, J. M., Hick, P. P., Jackson, B. V., Kuchar, T. A., and Price, S. D., "Measurement of the Gegenschein Brightness from the Solar Mass Ejection Imager (SMEI),” Icarus, 203, Sep 2009, pp. 124-133.

[84] Howard, R. A., Vourlidas, A., Korendyke, C. M., Plunkett, S. P., Carter, M. T., Wang, D., Rich, N., McMullin, D. R., Lynch, S., Thurn, A., Clifford, G., Socker, D. G., Thernisien, A. F., Chua, D., Linton, M. G., Keller, D., Janesick, J. R., Tower, J., Grygon, M., Hagood, R., Bast, W., Liewer, P. C., DeJong, E. M., Velli, M. M. C., Mikic, Z., Bothmer, V., Rochus, P., Halain, JP., and Lamy, P. L., "The Solar and Heliospheric Imager (SoloHI) Instrument for the Solar Orbiter Mission,” Proc. SPIE, 8862, Sep 2013, doi:10.1117/12.2027657.

[85] Vourlidas, A. Howard, R. A., Plunkett, S. P., Korendyke, C. M., Carter, M. T., Thernisien, A. F. R., Chua, D. H. Van Duyne, P., Socker, D. G., Linton, M. G., Liewer, P. C., Hall, J. R., Morrill, J. S., DeJong, E. M., Mikic, Z., Rochus, P. L. P. M., Bothmer, V., Rodman, J., and Lamy, P., "Seeing the Corona with the Solar Probe Plus Mission: The Wide-Field Imager for Solar Probe+ (WISPR),” Proc. SPIE, 8862, Sep 2013, doi:10.1117/12.2027508.

[86] Harrison, R., Davies, J., Davis, C., Eyles, C., and Hapgood, M., “Development and Application of a Heliospheric Imaging Mission," RAL Space final report, Rutherford Appleton Laboratory, Harwell Oxford, United Kingdom. 
[87] Howard, T. A., Bisi, M. M., Buffington, A, Clover, J. M., Cooke, M. P., Eyles, C. J., Hick, P. P., Holladay, P. E., Jackson, B. V., Johnston, J. C., Kahler, S. W., Kuchar, T. A., Mizuno, D. R., Penny, A. J., Price, S. D., Radick, R. R., Simnett, G. M., Tappin, S. J., Waltham, N. R., and Webb, D. F., “The Solar Mass Ejection Imager and Its Heliospheric Imaging Legacy,” Space Sci. Rev., 180, Dec 2013, pp. 1-38.

[88] Buffington, A., Hick, P. P., Jackson, B. V., and Korendyke, C. M., “Corrals, Hubcaps, and Crystal Balls: Some New Designs for Very-Wide-Angle Visible-Light Heliospheric Imagers,” Proc. SPIE, 3442, Nov 1998, pp. 77-86.

[89] Jackson, B. V., Buffington, A., Hick, P. P., Bisi, M. M., and Clover, J. M., “A Heliospheric Imager for Deep Space: Lessons Learned from Helios, SMEI, and STEREO,” Solar Phys., 265, Aug 2010, pp. 257-275.

[90] DeForest, C. E., Howard, T. A., and Tappin, S. J., “The Thomson Surface: Polarization,” Astrophys. J., 765, Mar 2013, doi:10.1088/0004-637X/765/44.

[91] Howard, T. A., “Three Dimensional Reconstruciton of Coronal Mass Ejections Using Heliospheric Imager Data,” J. Atmos. Solar. Terr. Phys., 73, Jun 2011, pp. 1242-1253.

[92] Webb, D. F., Biesecker, D. A., Gopalswamy, N., St. Cyr, O. C., Davila, J. M., Eyles, C. J., Thompson, B. J., Simunac, K. D. C., and Johnston, J. C., "Using STEREO-B as an L5 Space Weather Pathfinder Mission,” Space Res. Today, 178, Aug 2010, pp. 10-16.

[93] Gopalswamy, N., Davila, J. M., Auche`re, F., Schou, J., Korendyke, C., Shih, A., Johnston, J. C., MacDowall, R. J., Maksimovic, M., Sittler, E., Szabo, A., Wesenberg, R., Vennerstrom, S., and Heber, B., "Earth-Affecting Solar Causes Observatory (EASCO): A Mission at the Sun-Earth L5,” Proc. SPIE, 8148, 81480Z, Oct 2011, doi:10.1117/12.901538. 


\section{DISTRIBUTION LIST}

\section{DTIC/OCP}

8725 John J. Kingman Rd, Suite 0944

Ft Belvoir, VA 22060-6218

$1 \mathrm{cy}$

AFRL/RVIL

Kirtland AFB, NM 87117-5776

2 cys

Official Record Copy

AFRL/RVBXS/Dr. K. S. Balasubramaniam 1 cy 
This page is intentionally left blank. 